OPEN ACCESS

Edited by:

Jiawei Zhou

Institute of Neuroscience and Institutes for Biological Sciences

(CAS), China

Reviewed by:

Chen Haibo,

Beijing Hospital, China Dennis Qing Wang,

Third Affiliated Hospital of Sun Yat-sen

University, China

Peng Lei,

Sichuan University, China

*Correspondence:

Jun Liu

jly0520@hotmail.com

${ }^{\dagger}$ These authors have contributed equally to this work.

Received: 26 May 2017 Accepted: 04 September 2017 Published: 20 September 2017

Citation:

Li Y, Kang W, Zhang L, Zhou L, Niu M and Liu J (2017) Hyposmia Is Associated with RBD for PD Patients with Variants of SNCA. Front. Aging Neurosci. 9:303. doi: 10.3389/fnagi.2017.00303

\section{Hyposmia Is Associated with RBD for PD Patients with Variants of SNCA}

\author{
Yuanyuan $\mathrm{Li}^{\dagger}$, Wenyan Kang ${ }^{\dagger}$, Linyuan Zhang, Liche Zhou, Mengyue Niu and Jun Liu* \\ Department of Neurology, Institute of Neurology, Ruijin Hospital Affiliated to School of Medicine, Shanghai Jiaotong \\ University, Shanghai, China
}

Objective: Hyposmia may occur simultaneously with REM sleep behavior disorder $(\mathrm{RBD})$ as a specific phenotype in Parkinson's Diseases (PD), of which the disease progression is fast. In the study, we tried to identify whether the genotypic characteristics could participate in the co-occurrence of hyposmia and RBD in PD patients.

Methods: 152 PD patients were recruited from the Department of Neurology, Ruijin Hospital affiliated to Shanghai JiaoTong University School of Medicine from 2011 to 2016, with comprehensive clinical assessment performing. Two SNPs of SNCA (rs11931074 and rs894278) in 105 patients were also analyzed.

Results: Overall, 84 of 152 PD patients (55.3\%) were diagnosed with RBD after PSG evaluation. After regression analysis, higher levels of three parts of UPDRS and SCOPA-AUT scores were all associated with increased risk of RBD in PD patients, respectively. While for olfactory function, we didn't find significant correlation between hyposmia and RBD in PD patients. However, we found that in the group of minor G allele of rs894278, patients with lower score of SS-16 had a 4.76-fold risk of suffering from RBD in patients (95\% Cl: 1.39-16.67; $p=0.013)$. Furthermore, we analyzed SNP associated gene expression by eQTL analysis in Genevar database and found that GG genotype of rs894278 was associated with higher levels of $\alpha$-synuclein in Nerve tissue ( $p=1.5 \mathrm{E}-8$ ) while $\Pi$ genotype of $\mathrm{rs} 11931074$ was associated with higher levels of $\alpha$-synuclein in Brain $(p=0.0082)$, which suggesting a potential functional relevance with different symptoms of PD.

Conclusions: Hyposmia was associated with RBD in PD patients with the minor $\mathrm{G}$ allele of rs894278, which represent one specific subtype of PD. This study could provide more detail information about PD subtype of RBD with hyposmia in the future.

Keywords: Parkinson's disease, RBD, hyposmia, autonomic symptoms, single nucleotide polymorphisms

\section{INTRODUCTION}

Parkinson's disease (PD) is one of the most popular synucleinopathies characterized by core motor features, which often associated with non-motor manifestations, including sleeping disorders, olfactory loss, osteoporosis, personality changes, cognitive decline and autonomic abnormalities (Chaudhuri et al., 2006; Berg et al., 2015; Gao et al., 2015; Wei et al., 2016). Rapid eye movement sleep behavior disorder (RBD) is one of them, which is characterized by the loss of normal skeletal muscle atonia during REM sleep, accompanied with prominent motor activity in dream event (Boeve et al., 2007). Currently, one of the known strong identifiers of prognosis subtype is the 
co-occurrence of RBD and PD (Arnaldi et al., 2016). Previous study has been shown that RBD was associated with longer PD duration and higher dosage of dopaminergic therapy (LED), suggesting that the $\mathrm{PD}$ with $\mathrm{RBD}$ subtype may also have a different risk factor profile (Lee et al., 2010). Hyposmia is another common non-motor symptom, and 70-90\% PD patients suffer from olfactory impairment according to previous studies (Double et al., 2003).

Recently, It has been reported that olfactory dysfunction and RBD may occur simultaneously in some PD patients as a specific subtype of disease, in which patients were more likely to have akinetic-rigidity and cognitive dysfunction (Kang et al., 2016). However, the association between olfactory dysfunction and RBD in PD patients was not consistent (Jankovic et al., 2015; Arnaldi et al., 2016; Wan et al., 2016), at the same time, the underlying mechanism in RBD being co-occurrence with and hyposmia of PD patients was still not clear.

Previous studies found that carriers of Parkin and LRRK2 mutation more likely suffered from olfactory dysfunction (Khan et al., 2004, 2005), which is meaning that genotypic characteristics could affect the association between RBD and hyposmia in PD patients. We have demonstrated that hyposmia was correlates with SNCA variant in PD patients (Chen et al., 2015), so we performed the study to investigate whether the two typical SNPs of SNCA variants contribute to the co-occurrence hyposmia and $\mathrm{RBD}$ in $\mathrm{PD}$.

\section{METHODS}

\section{Participants}

All study participants were recruited from the Department of Neurology, Ruijin Hospital affiliated to Shanghai JiaoTong University School of Medicine from 2011 to 2016. 152 patients with Parkinson Disease (PD) were enrolled in our study with the diagnosis made according to the UK Brain Bank or current clinical diagnostic criteria by at least two movement disorders specialists (Hughes et al., 1992). Ages of the all participants were ranging from 50 to 80 years old, and patients with psychiatric disease or severe dementia were excluded. This study was approved by the ethics committee of Ruijin Hospital, Shanghai JiaoTong University School of Medicine, Shanghai, China, and all participants or their guardians provided written informed consent.

\section{Demographics and Clinical Assessment}

For these 152 PD patients, the disease stage was determined using the modified Hoehn and Yahr (HY) staging (range 0-5) (Hoehn and Yahr, 1967). The motor subscale of the Unified Parkinson's Disease Rating Scale (UPDRS II-III) was used to evaluate motor symptoms. Moreover, a series of comprehensive questionnaires besides the evaluating of motor symptoms

Abbreviations: PD, Parkinson's disease; iRBD, idiopathic rapid-eye-movement sleep behavior disorder; PSG, polysomnograph; SNP, single nucleotide polymorphisms; NMSQ, non-motor symptom quest; SCOPA-AUT, scale for outcomes in PD-Autonomic; HAMD-17, Hamilton Depression Rating Scale-17; MMSE, mini-mental state examination; eQTL, expression quantitative trait loci; OR, odds ratio. were also administered for every patient, including the nonmotor symptom quest (NMSQ), REM sleep behavior disorder screening questionnaire (RBDSQ), scale for outcomes in PDAutonomic (SCOPA-AUT), olfaction test (SS-16), Hamilton Depression Rating Scale-17 (HAMD-17) and Mini-Mental State Examination (MMSE). NMSQ was used to evaluate non-motor symptoms and SCOPA-AUT to assess autonomic dysfunction (Verbaan et al., 2007). An olfaction test (SS-16) was also performed to assess the olfactory function through 16-item odor identification (Hummel et al., 1997; Chen et al., 2012). HAMD-17 was administered to measure depressive symptoms (Hamilton, 1960), and MMSE was performed to evaluate the cognitive function resulting from PD (Zhang et al., 1990). Receiver operating characteristic (ROC) curves were plotted by calculating sensitivity and 1-specificity for specific cut-off values. Of the 152 PD patients, 84 were affected by RBD meeting the International Classification of Sleep Disorder (ISCD)-II criteria (American Academy of Sleep Medicine, 2005) and overnight video-polysomnograph (PSG) examination was done for each patient to confirm the diagnosis.

\section{Selection of SNPs and Genotyping}

Our previous studies were shown that SNCA rs894278 and rs11931074 were distributed in most of Chinese PD patients (Chen et al., 2015). So we measured the both SNPs in the recruited patients. Genomic DNA for 105 of the 152 PD patients was isolated from lymphocytes of whole blood using the QIAamp DNA extraction kit (Qiagen, Valencia, CA) and genetic information of was obtained by direct DNA sequencing performed on a 3730xl DNA analyzer (Applied Biosystems, Foster City, CA, USA) as we reported (Liu et al., 2013).

\section{Analysis of Expression Quantitative Trait Loci (eQTL), Functional Characterization and Mutation Frequency of the Two SNPs}

The potential functional impact of validated SNPs on gene expression was evaluated by analyzing gene-SNP association in expression quantitative trait loci (eQTL) studies with the Genevar database available in the HapMap3 dataset (Yang et al., 2010). While the predicted regulatory motif function of each SNP was predicted using ENCODE project explored from UCSC databased (http://www.broadinstitute.org/ mammals/haploreg/haploreg.php).

\section{Statistical Analysis}

Data were analyzed by the SPSS software version 20.0 (SPSS Inc., Chicago, IL, USA). Statistical significance was taken as two-sided $p<0.05$. Categorical variables were compared using the $\chi^{2}$ or Fisher exact test to assess the difference of baseline parameters between the PD with or without RBD group. Binary logistic regression analysis was used to determine the independent risk factors of RBD in PD patients and effect-size estimates were expressed as odds ratios (ORs) and 95\% confidence intervals (CIs). For statistical analysis between the subgroups, chi-square tests were used for categorical variables and students' tests for continuous variables. SNP analysis was performed by binary logistic regression analysis adjusting for age, sex, smoking and 
disease duration. The multivariable analysis was repeated for the replication patients.

\section{RESULTS}

\section{Demographic and Clinical Features}

The main clinical and demographic features of patients were listed in Table 1. Eighty four of 152 PD patients were affected by $\mathrm{RBD}$ (PD+RBD, 55.3\%). For these patients, gender proportion was difference: $79.8 \%$ were man in $\mathrm{PD}+\mathrm{RBD}$ group compared to $52.9 \%$ in PD without RBD group $(p<0.001)$, and patients with RBD presented longer disease duration and higher UPDRS (I-III) scores than $\mathrm{PD}$ without RBD group ( $p=0.046$ for PD duration; $p<0.001$ for UPDRS I; $p=0.002$ for UPDRS II; $p=0.017$ for UPDRS III). Moreover, there were more smokers $(p=0.041)$ and more patients suffering constipation in patients with RBD $(p=0.002$, Table 1).

\section{Analysis of Clinical Characteristics and SNPs between PD with or without RBD}

Due to the importance of RBD in PD disease, we further evaluated the clinical manifestation between $\mathrm{PD}$ with or without RBD. After logistic analysis, as shown in Table 2, patients with higher NMSQ scores were shown 11.7-fold (95\% CI: 4.4-31.5; $p<0.001)$ higher risk in $\mathrm{PD}+\mathrm{RBD}$ group. In terms of autonomic dysfunction, the odds of having worse syndromes in $\mathrm{PD}+\mathrm{RBD}$ patients were 6.1 times (95\% CI: $2.6-14.5 ; p<0.001$ ) risk. While for the influence of genetic characteristics, Table 3 was listed genotype distributions of rs894278, rs11931074 variants in the patients with and without RBD. After logistic regression analysis, adjusted by age, sex, smoking and PD duration, we didn't find significant different between gene variations and RBD occurrence in PD patients of dominant, recessive or additive model.

TABLE 1 | Demographic and clinical features in PD patients with or without RBD.

\begin{tabular}{lccr}
\hline & With RBD (n=84) & Without RBD (n=68) & $\boldsymbol{P}$ \\
\hline Age (years) & $64.2 \pm 7.4$ & $62.8 \pm 7.9$ & 0.267 \\
Sex & & & \\
$\quad$ Female & $17(20.2 \%)$ & $32(47.1 \%)$ & $<0.001$ \\
$\quad$ Male & $67(79.8 \%)$ & $36(52.9 \%)$ & \\
Smoking & & & 0.041 \\
$\quad$ Yes & $21(26.3 \%)$ & $8(12.5 \%)$ & \\
$\quad$ No & $59(73.8 \%)$ & $56(87.5 \%)$ & 0.002 \\
Constipation & & & \\
$\quad$ Yes & $41(64.1 \%)$ & $22(36.7 \%)$ & 0.046 \\
$\quad$ No & $23(35.9 \%)$ & $38(63.3 \%)$ & 0.096 \\
PD duration (years) & $4.7 \pm 4.1$ & $3.5 \pm 3.4$ & $<0.001$ \\
HY & $1.9 \pm 0.8$ & $1.7 \pm 0.8$ & 0.002 \\
UPDRS-I & $3.4 \pm 2.2$ & $1.9 \pm 1.7$ & 0.017 \\
UPDRS-II & $10.7 \pm 4.3$ & $8.1 \pm 4.3$ & $15.4 \pm 9.5$ \\
UPDRS-III & $19.4 \pm 10.4$ & & \\
\hline
\end{tabular}

$P D$, Parkinson's disease; RBD, REM sleep behavior disorder.

\section{Correlation between Hyposmia and RBD in PD Patients Classified by Gene Variants}

To verify whether genotypic characteristics would participate in the phenotype of hyposmia, we further evaluated the correlation between hyposmia and RBD in PD patients with different variants of rs894278 and rs11931074. The result indicated that PD patients with lower score of SS-16 had a 4.76 -fold risk of suffering from RBD in the minor G allele of rs894278 (95\% CI: $1.39-16.67 ; p=0.013$, Table 4). For the patients with the minor $\mathrm{G}$ allele of $\mathrm{rs} 894278,57.6 \%$ patients with SS-16 score below 8 exhibit RBD while only $23.1 \%$ didn't. As for the minor T allele of rs11931074, patients with decreased olfactory score were more incline to manifest RBD, but didn't achieve significant difference ( $p=0.082$, Table 4). 43.5\% PD patients with SS-16 score below 8 in the $\mathrm{T}$ allele of rs11931074 exhibit RBD while $16.7 \%$ not.

\section{Functional Prediction of rs894278 and rs11931074}

To fully understand the functional impacts of the identified SNPs of SNCA related to hyposmia and RBD, the SNP associated gene expression was tested by eQTL analysis in Genevar database (Figure 1). The results showed both SNPs were significantly associated with the gene expression in normal tissues: GG genotype of rs894278 was associated with higher levels of SNCA in Nerve ( $p=1.5 \mathrm{E}-8)$ while TT genotype of rs11931074 was associated with higher levels of SNCA in Brain $(p=0.0082)$. On the other hand, the potential impact of the two SNPs

TABLE 2 | Evaluation of the PD patients with or without RBD.

\begin{tabular}{|c|c|c|c|c|}
\hline & $\begin{array}{l}\text { With RBD } \\
(n=84)\end{array}$ & $\begin{array}{c}\text { Without } \\
\text { RBD }(n=68)\end{array}$ & OR (95\% Cl, adjusted) & $P$ \\
\hline \multicolumn{5}{|l|}{ NMSQ } \\
\hline$<8$ & 27 (32.1\%) & $50(84.7 \%)$ & 1 & \multirow[t]{2}{*}{$<0.001$} \\
\hline$\geq 8$ & 57 (67.9\%) & $9(15.3 \%)$ & $11.7(4.4-31.5)$ & \\
\hline \multicolumn{5}{|c|}{ SCOPA-AUT } \\
\hline$<9$ & $13(15.5 \%)$ & 38 (57.6\%) & 1 & \multirow[t]{2}{*}{$<0.001$} \\
\hline$\geq 9$ & $71(84.5 \%)$ & $28(42.4 \%)$ & $6.1(2.6-14.5)$ & \\
\hline \multicolumn{5}{|l|}{ SS-16 } \\
\hline$<8$ & 48 (58.5\%) & 30 (46.2\%) & 1 & \multirow[t]{2}{*}{0.566} \\
\hline$\geq 8$ & $34(41.5 \%)$ & 35 (53.8\%) & $0.8(0.4-1.8)$ & \\
\hline \multicolumn{5}{|c|}{ HAMD-17 } \\
\hline$<8$ & $42(50.6 \%)$ & 38 (56.7\%) & 1 & \multirow[t]{2}{*}{0.97} \\
\hline$\geq 8$ & $41(49.4 \%)$ & $29(43.3 \%)$ & $1.0(0.3-3.4)$ & \\
\hline \multicolumn{5}{|l|}{ MMSE } \\
\hline$<26$ & $14(17.3 \%)$ & $11(16.4 \%)$ & 1 & \multirow[t]{2}{*}{0.827} \\
\hline$\geq 26$ & $67(82.7 \%)$ & $56(83.6 \%)$ & $0.9(0.4-2.3)$ & \\
\hline \multicolumn{5}{|l|}{ LED } \\
\hline$<512.5$ & $44(73.3 \%)$ & $36(87.8 \%)$ & 1 & \multirow[t]{2}{*}{0.532} \\
\hline$\geq 512.5$ & $16(26.7 \%)$ & $5(12.2 \%)$ & $0.7(0.2-2.2)$ & \\
\hline
\end{tabular}

$P D$, Parkinson's disease; NMSQ, non-motor symptom quest; SCOPA-AUT, Scale for outcomes in PD-Autonomic; SS-16Q, Sniffin' Sticks 16 types tests; RBD, REM sleep behavior disorder; HAMD-17, Hamilton Depression Rating Scale-17; MMSE, Mini-Mental State Examination; LED, L-dopa Equivalent Dosage. All results were adjusted for age, sex, PD duration, constipation and smoking. OR, odd ratio; $95 \% \mathrm{Cl}, 95 \%$ confidence interval. 
TABLE 3 | Association of SNPs in PD patients with or without RBD.

\begin{tabular}{|c|c|c|c|c|c|c|c|c|}
\hline \multirow[t]{2}{*}{ SNP } & \multirow[t]{2}{*}{ Gene } & \multirow[t]{2}{*}{ Minor allele } & \multicolumn{2}{|c|}{ Dominant } & \multicolumn{2}{|c|}{ Recessive } & \multicolumn{2}{|c|}{ Additive } \\
\hline & & & OR (95\% Cl) & $\boldsymbol{P}$ & OR (95\% Cl) & $\boldsymbol{P}$ & OR $(95 \% \mathrm{Cl})$ & $\boldsymbol{P}$ \\
\hline rs894278 & SNCA & G & 0.87 (0.28-2.65) & 0.801 & $0.81(0.33-1.96)$ & 0.633 & $0.94(0.52-1.73)$ & 0.850 \\
\hline rs11931074 & SNCA & $\mathrm{T}$ & 2.19 (0.62-7.69) & 0.224 & $0.85(0.36-2.05)$ & 0.724 & $0.76(0.41-1.41)$ & 0.379 \\
\hline
\end{tabular}

All results were adjusted for age, sex, PD duration and smoking. OR, odd ratio; 95\% Cl, 95\% confidence interval.

TABLE 4 | Analysis of olfactory function in patients with or without gene variants of SNCA.

\begin{tabular}{|c|c|c|c|c|c|c|c|c|c|}
\hline & With RBD & Without RBD & OR (95\% Cl) & $P$ & & With RBD & Without RBD & OR (95\% Cl) & $P$ \\
\hline \multicolumn{10}{|c|}{ rs894278 } \\
\hline GG+ & & & & & $\Pi T$ & & & & \\
\hline$\geq 8$ & $14(42.4 \%)$ & 20 (76.9\%) & 1 & 0.013 & & 13 (50.0\%) & 12 (75.0\%) & 1 & 0.435 \\
\hline$<8$ & 19 (57.6\%) & $6(23.1 \%)$ & $4.76(1.39-16.67)$ & & & 13 (50.0\%) & $4(25.0 \%)$ & $1.82(0.40-8.33)$ & \\
\hline \multicolumn{10}{|c|}{ rs11931074 } \\
\hline$\pi+$ & & & & & $\mathrm{GG}$ & & & & \\
\hline$\geq 8$ & 13 (56.5\%) & 15 (83.3\%) & 1 & 0.082 & & 15 (41.7\%) & 17 (70.8\%) & 1 & 0.289 \\
\hline$<8$ & 10 (43.5\%) & 3 (16.7\%) & $4.17(0.83-20.0)$ & & & 21 (58.3\%) & 7 (29.2\%) & $1.96(0.56-6.67)$ & \\
\hline
\end{tabular}

RBD, REM sleep behavior disorder. All results were adjusted for age, sex, PD duration and smoking. OR, odd ratio; 95\% Cl, 95\% confidence interval.
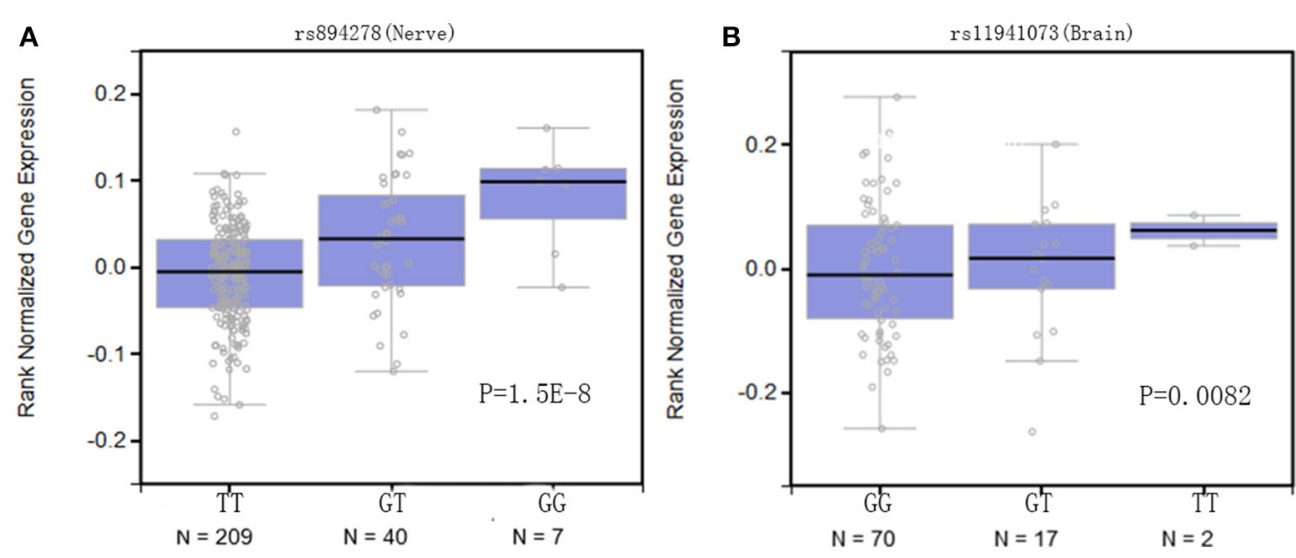

FIGURE 1 | Boxplots of SNCA expression from Genevar data. Below each boxplot is the number of individuals with each genotype. (A) GG carriers of rs894278 was associated with higher levels of SNCA in the Nerve $(p=1.5 \mathrm{E}-8)$; (B) TT genotype of rs11931074 was associated with higher levels of SNCA in the Brain ( $p=0.0082)$.

on gene function was analyzed using HaploReg. Based on bioinformatics analysis from the ENCODE project, the two SNPs were suggested to potentially affect transcription factor binding and have potential regulatory roles (Table 5), which rs894278 may be located in a predicted GCNF, Nanog_disc4, Nkx2_9, TATA_disc9, and Zfp410 motifs, and rs11931074 may be located in predicted SETDB1_disc1 motif. The above suggest that two SNPs of SNCA have potential functional relevance with PD.

\section{DISCUSSION}

To the best of our knowledge, the present study is the first to investigate the influence of genotypic characteristics on the association between RBD and hyposmia in PD patients. The most noteworthy finding was the identification of one promising SNP, SNCA rs894278, in which the patients harboring minor $\mathrm{G}$ allele was more likely to have hyposmia and RBD simultaneously, and may have worse disease progression.

Numerous studies have investigated many factors associated with the presence of RBD in PD patients. In our study, we found that $\mathrm{PD}+\mathrm{RBD}$ patients had male predominance and longer disease duration, which was in line with previous studies (Gjerstad et al., 2008; Yoritaka et al., 2009; Poryazova et al., 2013). Moreover, PD+RBD patients were presented with higher UPDRS (II-III) scores, which was consistent with previous study of factors related to RBD in 2010 (Lee et al., 2010) and study in 2014 
TABLE 5 | Functional prediction of SNPS using data from ENCODE.

\begin{tabular}{lllcr}
\hline Variables & Motifs $^{*}$ & Strand & Ref & Alt \\
\hline rs894278 & GCNF & - & -7 & 4.9 \\
& Nanog_disc4 & + & 10.6 & 12.9 \\
& Nkx2_9 & + & 11.9 & 14.7 \\
& TATA_disc9 & - & 0.8 & 12.8 \\
& Zfp410 & - & 11.4 & 10.4 \\
rs11931074 & SETDB1_disc1 & + & 9 & 8.4 \\
\hline
\end{tabular}

ENCODE, the Encyclopedia of DNA Elements; *Evidence of alteration in regulatory motif.

(Gong et al., 2014). Furthermore, after risk regression analysis of developing $\mathrm{RBD}$, several non-motor symptoms appeared increasing incidence. Previous studies have shown autonomic dysfunction including autonomic symptom scores assessing, formal autonomic testing and cardiac MIBG scintigraphy have been related to idiopathic RBD, as also found in this study (Miyamoto et al., 2006; Postuma et al., 2008; Yoritaka et al., 2009). As for olfactory function, hyposmia didn't appear to be a risk factor for RBD of PD patients in our study, which was in line with one previous study of 2015 (Jankovic et al., 2015). However, there are also studies showed that hyposmia may simultaneously occurred with RBD as a specific subtype of $\mathrm{PD}$, in which patients were more likely to suffer from cognitive dysfunction and exhibit akinetic-rigid-type (Kang et al., 2016).

Previous studies have shown that PD have different genetic heterogeneity and phenotypic imprecision. A-synuclein (SNCA) is a hallmark of PD and crucial factor in PD pathogenesis. Clinical-pathological studies have also suggested that both hyposmia and RBD may be related to a-synuclein deposition (Ubeda-Banon et al., 2010). On the other hand, we have demonstrated that hyposmia was correlates with SNCA variants in PD patients in our previous study (Chen et al., 2015), thus it is worth exploring whether these variants are associated with the specific subtype in PD. Rs894278 and rs11931074 SNPs in SNCA has been identified as conferring the strongest PD risk (Liu et al., 2013) and recently, studies have shown these two SNPs were also associated with some other phenotypes in PD, such as festination (Zheng et al., 2017) and earlier onset of PD (Huang et al., 2015). So, we further evaluated these two SNPs to identify the relationship, and the result indicated that hyposmia were associated with RBD in PD patients with minor $G$ allele of rs894278, which means PD patients harboring this allele was more likely to have hyposmia and RBD simultaneously and worse disease progression.

On the other hand, functional characterization might be important to dissect the mechanistic basis for the variants' association with clinical subtypes, thus we explored the gene expression by eQTL analysis in Genevar public database. And

\section{REFERENCES}

American Academy of Sleep Medicine (2005). ICSD-2-International Classification of Sleep Disorders, 2nd Edn., Diagnostic and Coding Manual. Westchester, IL: American Academy of Sleep Medicine. the result was shown that the genotypes of two SNPs were significantly correlated with expression of $\alpha$-synuclein in tissues or cell lines, which suggested a potential functional relevance with PD. The study with clinical and genetic information would help us to explore the progression and precision management for a subtype of RBD with olfactory dysfunction in PD patients. Further studies on the mechanism of how these variants influenced the pathogenesis and progression of PD are needed in the future study.

In conclusion, we found that $\mathrm{PD}$ with $\mathrm{RBD}$ patients had higher scores in UPDRS three parts, and more NMS and autonomic dysfunctions, especially constipation. Moreover, patients with hyposmia were co-occurrence with RBD in the minor G allele of rs894278, which would present one specific subtype of PD.

\section{ETHICS STATEMENT}

This study was carried out in accordance with the recommendations of the "ethics committee of Ruijin Hospital, Shanghai JiaoTong University School of Medicine, Shanghai, China" with written informed consent from all subjects. All subjects gave written informed consent in accordance with the Declaration of Helsinki. The protocol was approved by the "ethics committee of Ruijin Hospital, Shanghai JiaoTong University School of Medicine, Shanghai, China."

\section{AUTHOR CONTRIBUTIONS}

JL conceived, supervised the project and contributed to patients' recruitment. YL, JL, and WK drafted the manuscript. YL, WK, LZha, LZho, and MN contributed to sample collection, performed data management and statistical analyses. All authors read and approved the final version of the manuscript.

\section{FUNDING}

This work was supported by grants from the National Natural Science Foundation of China (81471287, 81071024, 81171202, 81371407, 30872729, and 30870879), the National Key Research and Development Program (2016YFC1306505), the Shanghai Municipal Education Commission-Gaofeng Clinical Medicine Grant (20152201), the Innovation Fund of Shanghai JiaoTong University School of Medicine (BXJ201715).

\section{ACKNOWLEDGMENTS}

The authors thank YL, WK, LZha, LZho, MN, and JL for study coordination. We acknowledge all the subjects for their generous donations of saliva samples. 
Berg, D., Postuma, R. B., Adler, C. H., Stern, M., Poewe, W., Olanow, C. W., et al. (2015). MDS research criteria for prodromal Parkinson's disease. Mov. Disord. 30, 1600-1611. doi: 10.1002/mds.26431

Boeve, B. F., Silber, M. H., Saper, C. B., Ferman, T. J., Dickson, D. W., Parisi, J. E., et al. (2007). Pathophysiology of REM sleep behaviour disorder and relevance to neurodegenerative disease. Brain 130, 2770-2788. doi: 10.1093/brain/awm056

Chaudhuri, K. R., Healy, D. G., and Schapira, A. H. (2006). National institute for clinical E. non-motor symptoms of Parkinson's disease: diagnosis and management. Lancet Neurol. 5, 235-245. doi: 10.1016/S1474-4422(06)70373-8

Chen, W., Chen, S., Kang, W. Y., Li, B., Xu, Z. M., Xiao, Q., et al. (2012). Application of odor identification test in Parkinson's disease in China: a matched case-control study. J. Neurol. Sci. 316, 47-50. doi: 10.1016/j.jns.2012.01.033

Chen, W., Kang, W. Y., Chen, S., Wang, Y., Xiao, Q., Wang, G., et al. (2015). Hyposmia correlates with SNCA variant and non-motor symptoms in Chinese patients with Parkinson's disease. Parkins. Relat. Disord. 21, 610-614. doi: 10.1016/j.parkreldis.2015.03.021

Double, K. L., Rowe, D. B., Hayes, M., Chan, D. K., Blackie, J., Corbett, A., et al. (2003). Identifying the pattern of olfactory deficits in Parkinson disease using the brief smell identification test. Arch. Neurol. 60, 545-549. doi: 10.1001/archneur.60.4.545

Gao, H., Wei, X., Liao, J., Wang, R., Xu, J., Liu, X., et al. (2015). Lower Bone mineral density in patients with Parkinson's disease: a cross-sectional study from chinese mainland. Front. Aging Neurosci. 7:203. doi: 10.3389/fnagi.2015. 00203

Gjerstad, M. D., Boeve, B., Wentzel-Larsen, T., Aarsland, D., and Larsen, J. P. (2008). Occurrence and clinical correlates of REM sleep behaviour disorder in patients with Parkinson's disease over time. J. Neurol. Neurosurg. Psychiatr. 79, 387-391. doi: 10.1136/jnnp.2007.116830

Gong, Y., Xiong, K. P., Mao, C. J., Shen, Y., Hu, W. D., Huang, J. Y., et al. (2014). Clinical manifestations of Parkinson disease and the onset of rapid eye movement sleep behavior disorder. Sleep Med. 15, 647-653. doi: 10.1016/j.sleep.2013.12.021

Hamilton, M. (1960). A rating scale for depression. J. Neurol. Neurosurg. Psychiatr. 23, 56-62. doi: 10.1136/jnnp.23.1.56

Hoehn, M. M., and Yahr, M. D. (1967). Parkinsonism: onset, progression, and mortality. Neurology 57, S11-2S6. doi: 10.1212/WNL.17.5.427

Huang, Y., Wang, G., Rowe, D., Wang, Y., Kwok, J. B. J., Xiao, Q., et al. (2015). SNCA gene, but not MAPT, influences onset age of Parkinson's disease in Chinese and Australians. Biomed. Res. Int. 2015:135674. doi: 10.1155/2015/135674

Hughes, A. J., Daniel, S. E., Kilford, L., and Lees, A. J. (1992). Accuracy of clinical diagnosis of idiopathic Parkinson's disease: a clinico-pathological study of 100 cases. J. Neurol. Neurosurg. Psychiatr. 55, 181-184. doi: 10.1136/jnnp.55.3.181

Hummel, T., Sekinger, B., Wolf, S. R., Pauli, E., and Kobal, G. (1997). 'Sniffin' sticks': olfactory performance assessed by the combined testing of odor identification, odor discrimination and olfactory threshold. Chem. Senses 22, 39-52. doi: 10.1093/chemse/22.1.39

Jankovic, M., Svetel, M., and Kostic, V. (2015). Frequency of REM sleep behavior disorders in patients with Parkinson's disease. Vojnosanit. Pregl. 72, 442-446. doi: 10.2298/VSP130501006J

Kang, S. H., Lee, H. M., Seo, W. K., Kim, J. H., and Koh, S. B. (2016). The combined effect of REM sleep behavior disorder and hyposmia on cognition and motor phenotype in Parkinson's disease. J. Neurol. Sci. 368, 374-378. doi: 10.1016/j.jns.2016.07.057

Khan, N. L., Jain, S., Lynch, J. M., Pavese, N., Abou-Sleiman, P., Holton, J. L., et al. (2005). Mutations in the gene LRRK2 encoding dardarin (PARK8) cause familial Parkinson's disease: clinical, pathological, olfactory and functional imaging and genetic data. Brain 128, 2786-2796. doi: 10.1093/brain/awh667
Khan, N. L., Katzenschlager, R., Watt, H., Bhatia, K. P., Wood, N. W., Quinn, N., et al. (2004). Olfaction differentiates parkin disease from early-onset parkinsonism and Parkinson disease. Neurology 62, 1224-1226. doi: 10.1212/01.WNL.0000118281.66802.81

Lee, J. E., Kim, K. S., Shin, H. W., and Sohn, Y. H. (2010). Factors related to clinically probable REM sleep behavior disorder in Parkinson disease. Parkinsonism Relat. Disord. 16, 105-108. doi: 10.1016/j.parkreldis.2009.08.005

Liu, J., Xiao, Q., Wang, Y., Xu, Z. M., Wang, Y., Yang, Q., et al. (2013). Analysis of genome-wide association study-linked loci in Parkinson's disease of Mainland China. Mov. Disord. 28, 1892-1895. doi: 10.1002/mds.25599

Miyamoto, T., Miyamoto, M., Inoue, Y., Usui, Y., Suzuki, K., and Hirata, K. (2006). Reduced cardiac 123I-MIBG scintigraphy in idiopathic REM sleep behavior disorder. Neurology 67, 2236-2238. doi: 10.1212/01.wnl.0000249313.25627.2e

Poryazova, R., Oberholzer, M., Baumann, C. R., and Bassetti, C. L. (2013). REM sleep behavior disorder in Parkinson's disease: a questionnaire-based survey. J. Clin. Sleep Med. 9, 55-59. doi: 10.5664/jcsm.2340

Postuma, R. B., Gagnon, J. F., Vendette, M., Charland, K., and Montplaisir, J. (2008). Manifestations of Parkinson disease differ in association with REM sleep behavior disorder. Mov. Disord. 23, 1665-1672. doi: 10.1002/mds.22099

Ubeda-Banon, I., Saiz-Sanchez, D., de la Rosa-Prieto, C., Argandona-Palacios, L., Garcia-Munozguren, S., and Martinez-Marcos, A. (2010). alphaSynucleinopathy in the human olfactory system in Parkinson's disease: involvement of calcium-binding protein- and substance P-positive cells. Acta Neuropathol. 119, 723-735. doi: 10.1007/s00401-010-0687-9

Verbaan, D., Marinus, J., Visser, M., van Rooden, S. M., Stiggelbout, A. M., and van Hilten, J. J. (2007). Patient-reported autonomic symptoms in Parkinson disease. Neurology 69, 333-341. doi: 10.1212/01.wnl.0000266593.50534.e8

Wan, Y., Luo, Y., Gan, J., Hu, R., Zhou, M., and Liu, Z. (2016). Clinical markers of neurodegeneration in Chinese patients with idiopathic rapid eye movement sleep behavior disorder. Clin. Neurol. Neurosurg. 150, 105-109. doi: 10.1016/j.clineuro.2016.09.002

Wei, X. B., Yan, R. H., Chen, Z. Y., Weng, R., Liu, X., Gao, H., et al. (2016). Combined diffusion tensor imaging and arterial spin labeling as markers of early Parkinson's disease. Sci. Rep. 6:33762. doi: 10.1038/srep33762

Yang, T. P., Beazley, C., Montgomery, S. B., Dimas, A. S., Gutierrez-Arcelus, M., Stranger, B. E., et al. (2010). Genevar: a database and Java application for the analysis and visualization of SNP-gene associations in eQTL studies. Bioinformatics 26, 2474-2476. doi: 10.1093/bioinformatics/btq452

Yoritaka, A., Ohizumi, H., Tanaka, S., and Hattori, N. (2009). Parkinson's disease with and without REM sleep behaviour disorder: are there any clinical differences? Eur. Neurol. 61, 164-170. doi: 10.1159/000189269

Zhang, M. Y., Katzman, R., Salmon, D., Jin, H., Cai, G. J., Wang, Z. Y., et al. (1990). The prevalence of dementia and Alzheimer's disease in Shanghai, China: impact of age, gender, and education. Ann. Neurol. 27, 428-437. doi: 10.1002/ana.410270412

Zheng, J. H., Yang, X. L., Zhao, Q. Z., Tian, S., Huang, H., Chen, Y., et al. (2017). Festination correlates with SNCA polymorphism in Chinese patients with Parkinson's disease. Parkinsons Dis. 2017:3176805. doi: 10.1155/2017/3176805

Conflict of Interest Statement: The authors declare that the research was conducted in the absence of any commercial or financial relationships that could be construed as a potential conflict of interest.

Copyright (c) 2017 Li, Kang, Zhang, Zhou, Niu and Liu. This is an open-access article distributed under the terms of the Creative Commons Attribution License (CC BY). The use, distribution or reproduction in other forums is permitted, provided the original author(s) or licensor are credited and that the original publication in this journal is cited, in accordance with accepted academic practice. No use, distribution or reproduction is permitted which does not comply with these terms. 